\title{
Euler-Lagrange Elasticity with Dynamics
}

\author{
H. H. Hardy \\ Math and Physics Department, Piedmont College, Demorest, GA, USA \\ Email: hhardy@piedmont.edu
}

Received 10 October 2014; revised 12 November 2014; accepted 19 November 2014

Copyright (C) 2014 by author and Scientific Research Publishing Inc.

This work is licensed under the Creative Commons Attribution International License (CC BY).

http://creativecommons.org/licenses/by/4.0/

c) (i) Open Access

\begin{abstract}
The equations of Euler-Lagrange elasticity describe elastic deformations without reference to stress or strain. These equations as previously published are applicable only to quasi-static deformations. This paper extends these equations to include time dependent deformations. To accomplish this, an appropriate Lagrangian is defined and an extrema of the integral of this Lagrangian over the original material volume and time is found. The result is a set of Euler equations for the dynamics of elastic materials without stress or strain, which are appropriate for both finite and infinitesimal deformations of both isotropic and anisotropic materials. Finally, the resulting equations are shown to be no more than Newton's Laws applied to each infinitesimal volume of the material.
\end{abstract}

\section{Keywords}

Elasticity, Stress, Strain, Infinitesimal Deformations, Finite Deformations, Discrete Region Model

\section{Background}

Virtually all modern theories of elasticity [1]-[4] build the equations to describe elasticity using stress and/or strain. Hardy [5] proposed to return to the approach of Euler, Lagrange, and Poisson [6] to build the equations of elasticity using point locations and forces instead of stress and strain. Hardy called these equations the equations of Euler-Lagrange elasticity. The equations of Euler-Lagrange elasticity are appropriate for quasi-static deformations, but do not include dynamics. Dynamics will be added in this paper.

Hardy defined an elastic material as one which when deformed, stores energy; and when it is returned to its original state, the stored energy is returned to its surroundings. This is known as hyper-elasticity [7]. Hardy followed the notation of Spencer [8] by defining the initial position of each point in an elastic material to be $X_{1}$, $X_{2}$, and $X_{3}$ corresponding to the $x, y$, and $z$ coordinates of that point. The parameters, $x_{1}, x_{2}, x_{3}$ were defined as the $x, y, z$ coordinates of the corresponding point after the deformation. The final position of each point depends upon the initial position, so that each component of each point, $x_{i}$, is a function of $X_{1}, X_{2}$, 
and $X_{3}$. The energy of the material is a function of the final positions of each point $x_{i}(i=1,2,3)$ and the relative change in distances between points, $\frac{\partial x_{i}}{\partial X_{j}}$ (i and $\left.j=1,2,3\right)$. This energy is expressed in terms of the energy per unit original volume, $E\left(x_{i}, \frac{\partial x_{i}}{\partial X_{j}}\right)$, which can be divided into the energy associated with body forces, $E_{\text {body }}\left(x_{i}\right)$, plus the energy associated with the deformation of the body, $E_{\text {def }}\left(\frac{\partial x_{i}}{\partial X_{j}}\right)$,

$$
E=E_{\text {body }}\left(x_{i}\right)+E_{\text {def }}\left(\frac{\partial x_{i}}{\partial X_{j}}\right)
$$

To obtain the Euler-Lagrange differential equations, Hardy minimized the total energy, $\mathcal{E}_{\text {tot }}$,

$$
\delta \mathcal{E}_{\text {tot }}=\delta \iiint E\left(x_{i}, \frac{\partial x_{i}}{\partial X_{j}}\right) \mathrm{d} X_{1} \mathrm{~d} X_{1} \mathrm{~d} X_{3}=0,
$$

which resulted in three Euler equations,

$$
\frac{\partial E}{\partial x_{i}}-\frac{\mathrm{d}}{\mathrm{d} X_{j}} \frac{\partial E}{\partial\left(\partial x_{i} / \partial X_{j}\right)}=0 \text { for } i=1,2,3 .
$$

The advantage of Hardy's approach is that Equation (3) is applicable to both infinitesimal and finite deformations as well as being appropriate for both anisotropic and isotropic materials. The disadvantage of this approach is that it is only appropriate for quasi-static deformations, since time dependence is not included. In this paper, I will extend this approach to include dynamics.

\section{Adding Dynamics}

To add dynamics to the Euler-Lagrange elasticity equations several changes are needed to the quasi-static approach. First define each $x_{i}$ as a function of time as well as $X_{1}, X_{2}$, and $X_{3}$. Second define an appropriate Lagrangian. Third minimize the integral of the Lagrangian over both space and time. Lagrangians for particle dynamics are defined as the kinetic energy minus the potential energy of the particle. To extend this to a distributed material, our "particle" will be an infinitesimal volume of the elastic material. Define the kinetic energy per original volume of the material as

$$
T=\frac{1}{2} \rho v^{2},
$$

with $\rho$ the mass per original volume of the material and the velocity of any point in the material, $v$, is

$$
v^{2}=\left(\frac{\partial x_{1}}{\partial t}\right)^{2}+\left(\frac{\partial x_{2}}{\partial t}\right)^{2}+\left(\frac{\partial x_{3}}{\partial t}\right)^{2} .
$$

Define the potential energy per unit original volume as $E$ in Equation (1) and the Lagrangian, $\mathcal{L}$ as

$$
\mathcal{L}=T-E \text {. }
$$

Substitute Equation (1) into Equation (6) with $E_{\text {body }}=\rho g x_{3}$ and $T$ from Equation (4) to express $\mathcal{L}$ as

$$
\mathcal{L}=\frac{1}{2} \rho\left(\left(\frac{\partial x_{1}}{\partial t}\right)^{2}+\left(\frac{\partial x_{2}}{\partial t}\right)^{2}+\left(\frac{\partial x_{3}}{\partial t}\right)^{2}\right)-\rho g x_{3}-E_{\text {def }}\left(\frac{\partial x_{i}}{\partial X_{j}}\right)
$$

Now find the extrema of

$$
\mathcal{M}=\iiint \int \mathcal{L} \mathrm{d} X_{1} \mathrm{~d} X_{2} \mathrm{~d} X_{3} \mathrm{~d} t
$$


Since $\mathcal{L}=f\left(x_{i}, \frac{\partial x_{i}}{\partial X_{j}}, \frac{\partial x_{i}}{\partial t}\right)$, the following three Euler equations result from setting $\delta \mathcal{M}=0$ :

$$
\frac{\partial \mathcal{L}}{\partial x_{i}}-\frac{\mathrm{d}}{\mathrm{d} X_{j}} \frac{\partial \mathcal{L}}{\partial\left(\partial x_{i} / \partial X_{j}\right)}-\frac{\mathrm{d}}{\mathrm{d} t} \frac{\partial \mathcal{L}}{\partial\left(\partial x_{i} / \partial t\right)}=0
$$

Substituting $\mathcal{L}$ from Equation (7) gives

$$
-\rho g \delta_{i 3}+\frac{\mathrm{d}}{\mathrm{d} X_{j}}\left(\frac{\partial E_{d e f}}{\partial\left(\partial x_{i} / \partial X_{j}\right)}\right)-\frac{\mathrm{d}}{\mathrm{d} t}\left(\rho \frac{\partial x_{i}}{\partial t}\right)=0
$$

or

$$
\frac{\mathrm{d}}{\mathrm{d} t}\left(\rho \frac{\partial x_{i}}{\partial t}\right)=-\rho g \delta_{i 3}+\frac{\mathrm{d}}{\mathrm{d} X_{j}}\left(\frac{\partial E_{d e f}}{\partial\left(\partial x_{i} / \partial X_{j}\right)}\right) .
$$

Equation (11) are the equations of dynamics for deformation of elastic materials. All that is required is to define $E_{\text {def }}$ of the material experimentally. The $E_{\text {def }}$ must be invariant under coordinate rotations and translations. One method is to define $E_{\text {def }}$ in terms of invariants of the $\frac{\partial x_{i}}{\partial X_{j}}$ matrix (e.g. Ogden [9], Hardy [10]).

Note that no assumptions of infinitesimal deformation or isotropy have been made to derive Equation (11), so they are applicable for both infinitesimal and finite deformations of both isotropic and anisotropic materials. The most surprising thing about Equation (11) is that each term in Equation (11) can be given a simple physical interpretation.

\section{Physical Interpretation of the Terms in Equation (11)}

In order to give a physical interpretation to the individual terms in Equation (11) consider a small cuboid defined as $\Delta X_{1} \Delta X_{2} \Delta X_{3}$. The term on the left hand side of Equation (11), $\frac{\mathrm{d}}{\mathrm{d} t}\left(\rho \frac{\partial x_{i}}{\partial t}\right)$, is the change in momentum per unit original volume of this cuboid with respect to time in the limit as $\Delta X_{1}, \Delta X_{2}$ and $\Delta X_{3}$ approach 0 . The first term on the right hand side, $-\rho g \delta_{i 3}$, is the force of gravity per unit original volume of this cuboid in the same limit. The second term on the right hand side, $\frac{\mathrm{d}}{\mathrm{d} X_{j}}\left(\frac{\partial E_{\text {def }}}{\partial\left(\partial x_{i} / \partial X_{j}\right)}\right)$, is shown below to be the net surface force per unit original volume applied to all the surfaces of the cuboid as the volume of the cuboid shrinks to zero. In other words, Equation (11) is just an expression of Newton's laws $\left(\sum \boldsymbol{F}=\frac{\mathrm{d} \boldsymbol{p}}{\mathrm{d} t}\right)$ for each infinitesimal volume of the material.

To see that $\frac{\mathrm{d}}{\mathrm{d} X_{j}}\left(\frac{\partial E_{\text {def }}}{\partial\left(\partial x_{i} / \partial X_{j}\right)}\right)$ is indeed the net surface force per unit original volume acting on the cuboid, recall that Hardy [5] found that the external force acting on a surface can be written as

$$
\mathrm{d} F_{i}=\frac{\partial E_{d e f}}{\partial\left(\partial x_{i} / \partial X_{j}\right)} \mathrm{d} A_{j} .
$$

Let $\mathrm{d} \boldsymbol{a}$ represent a particular plane during deformation, where the magnitude of $\mathrm{d} \boldsymbol{a}$ is the current infinitesimal area of the plane and the direction of $\mathrm{d} \boldsymbol{a}$ is perpendicular to the plane of interest and pointing away from the material receiving the force. To calculate the force on this plane using Equation (12), find the original magnitude and direction of $\mathrm{d} \boldsymbol{a}$ before the deformation. Call this $\mathrm{d} \boldsymbol{A}$. Define the components of $\mathrm{d} \boldsymbol{A}$ 
be $\mathrm{d} A_{1}, \mathrm{~d} A_{2}$, and $\mathrm{d} A_{3}$ in the $X_{1}, X_{2}$, and $X_{3}$ directions respectively. The three components of the force exerted on the $\mathrm{d} \boldsymbol{a}$ plane at any time during the deformation are then calculated from Equation (12) as

$$
\mathrm{d} F_{i}=\frac{\partial E_{\text {def }}}{\partial\left(\partial x_{i} / \partial X_{1}\right)} \mathrm{d} A_{1}+\frac{\partial E_{\text {def }}}{\partial\left(\partial x_{i} / \partial X_{2}\right)} \mathrm{d} A_{2}+\frac{\partial E_{\text {def }}}{\partial\left(\partial x_{i} / \partial X_{3}\right)} \mathrm{d} A_{3} .
$$

For our cuboid, defined as $\Delta X_{1} \Delta X_{2} \Delta X_{3}$, the $i^{\text {th }}$ component of the force on a plane of the cuboid originally perpendicular to $X_{j}$ is $F_{i j}$, where

$$
\left.F_{i j}=\frac{\partial E_{d e f}}{\partial\left(\partial x_{i} / \partial X_{j}\right)} \mathrm{d} A_{j} \text { (not summed over } j\right) .
$$

For example, $F_{33}$ is the $X_{3}$ component of the force on plane $\mathrm{d} A_{3}=\mathrm{d} X_{1} \mathrm{~d} X_{2}$. Divide the body into cuboids along the $X_{3}$ direction as shown in Figure 1(a). As shown in this figure, $F_{33}\left(X_{3}\right)$ is the component of force on region a from region $\mathrm{b}$ in the $X_{3}$ direction. $F_{33}\left(X_{3}+\Delta X_{3}\right)$ is the component of force on region $\mathrm{b}$ from region c. If we wish to express the net force on region $\mathrm{b}$ alone, this would be $F_{33}\left(X_{3}+\Delta X_{3}\right)-F_{33}\left(X_{3}\right)$ as shown in Figure 1(b). The net force in the $X_{3}$ direction on region b along the $X_{3}$ direction when divided by the cuboid's original volume is

$$
\begin{aligned}
\frac{F_{33}\left(X_{3}+\Delta X_{3}\right)-F_{33}\left(X_{3}\right)}{\Delta X_{1} \Delta X_{2} \Delta X_{3}} & =\frac{\left(\frac{\partial E_{\text {def }}}{\partial\left(\partial X_{3} / \partial X_{3}\right)}\right)_{X_{1}, X_{2}, X_{3}+\Delta X_{3}} \Delta X_{1} \Delta X_{2}}{\Delta X_{1} \Delta X_{2} \Delta X_{3}}-\frac{\left(\frac{\partial E_{\text {def }}}{\partial\left(\partial X_{3} / \partial X_{3}\right)}\right)_{X_{1}, X_{2}, X_{3}} \Delta X_{1} \Delta X_{2}}{\Delta X_{1} \Delta X_{2} \Delta X_{3}} \\
& =\frac{\left(\frac{\partial E_{\text {def }}}{\partial\left(\partial X_{3} / \partial X_{3}\right)}\right)_{X_{1}, X_{2}, X_{3}+\Delta X_{3}}-\left(\frac{\partial E_{\text {def }}}{\partial\left(\partial X_{3} / \partial X_{3}\right)}\right)_{X_{1}, X_{2}, X_{3}}}{\Delta X_{3}} .
\end{aligned}
$$

Taking the limit as the dimensions of the cube go to zero gives the net force per unit original volume on region $\mathrm{b}$ in the $X_{3}$ direction on the $\mathrm{d} A_{3}$ faces of the cube, $F_{33}^{\text {net }}$, to be

$$
\begin{aligned}
F_{33}^{\text {net }} & =\lim _{\Delta X_{1}, \Delta X_{2}, \Delta X_{3} \rightarrow 0} \frac{F_{33}\left(X_{3}+\Delta X_{3}\right)-F_{33}\left(X_{3}\right)}{\Delta X_{1} \Delta X_{2} \Delta X_{3}} \\
& =\lim _{\Delta X_{3} \rightarrow 0} \frac{\left(\frac{\partial E_{d e f}}{\partial\left(\partial x_{3} / \partial X_{3}\right)}\right)_{X_{1}, X_{2}, X_{3}+\Delta X_{3}}-\left(\frac{\partial E_{\text {def }}}{\partial\left(\partial X_{3} / \partial X_{3}\right)}\right)_{X_{1}, X_{2}, X_{3}}}{\Delta X_{3}} \\
& =\frac{\mathrm{d}}{\mathrm{d} X_{3}} \frac{\partial E_{\text {def }}}{\partial\left(\partial x_{3} / \partial X_{3}\right)} .
\end{aligned}
$$

A similar argument using $F_{11}$ and $F_{22}$ yields the net forces normal to the $\mathrm{d} A_{1}$ and $\mathrm{d} A_{2}$ faces, $F_{11}^{\text {net }}$ and $F_{22}^{\text {net }}$, to be

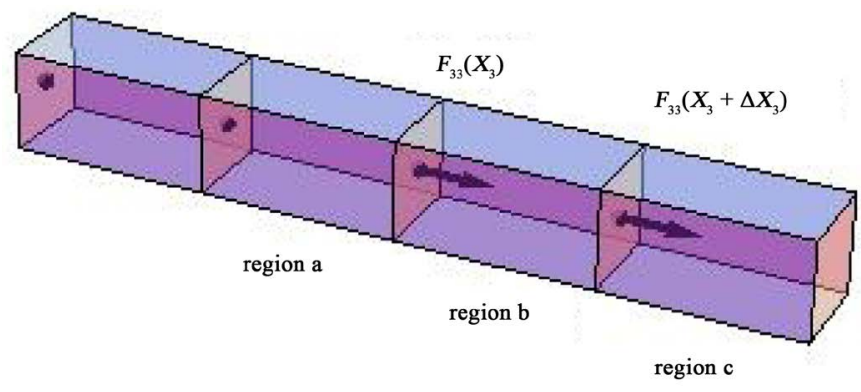

(a)

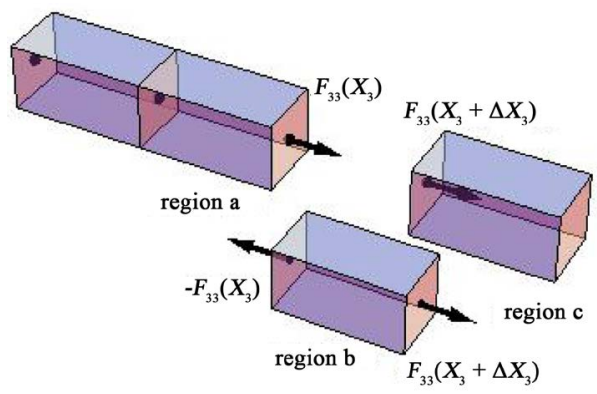

(b)

Figure 1. Force within the material in the $X_{3}$ direction on the $\mathrm{d} A_{3}$ surfaces (a) internal forces from Equation (14) (b) forces on region $b$. 


$$
F_{11}^{n e t}=\frac{\mathrm{d}}{\mathrm{d} X_{1}} \frac{\partial E_{d e f}}{\partial\left(\partial x_{1} / \partial X_{1}\right)}
$$

and

$$
F_{22}^{n e t}=\frac{\mathrm{d}}{\mathrm{d} X_{2}} \frac{\partial E_{d e f}}{\partial\left(\partial x_{2} / \partial X_{2}\right)} .
$$

Next consider $F_{32}^{\text {net }}$. Using Figure 2 and an argument similar to the one used in Figure 1 gives

$$
F_{32}^{\text {net }}=\frac{\mathrm{d}}{\mathrm{d} X_{2}} \frac{\partial E_{\mathrm{def}}}{\partial\left(\partial x_{3} / \partial X_{2}\right)},
$$

and in general

$$
F_{i j}^{n e t}=\frac{\mathrm{d}}{\mathrm{d} X_{j}} \frac{\partial E_{d e f}}{\partial\left(\partial x_{i} / \partial X_{j}\right)} \quad(\text { not summed over } j) .
$$

Combining these results, we have the total force in the $X_{i}$ direction to be

$$
F_{i}^{n e t}=\frac{\mathrm{d}}{\mathrm{d} X_{j}}\left(\frac{\partial E_{d e f}}{\partial\left(\partial x_{i} / \partial X_{j}\right)}\right),
$$

for $i=1,2,3$, and summed over $j=1,2,3$, which is the third term in Equation (11). Thus $\frac{\mathrm{d}}{\mathrm{d} X_{j}}\left(\frac{\partial E_{d e f}}{\partial\left(\partial x_{i} / \partial X_{j}\right)}\right)$ is the net surface force per unit original volume in the $i^{\text {th }}$ direction on any cuboid in the limit as the cuboid dimensions shrink to zero.

Figure 3 summarizes this result by illustrating the forces summed in each direction to calculate the net surface force on a cuboid of material. Note that in Figure 3 only the forces on the "front" faces of the cuboid are

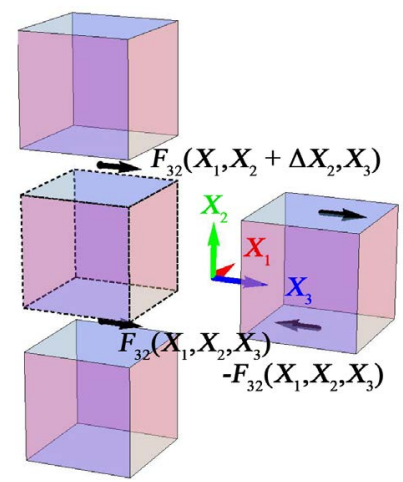

Figure 2. Forces in the $X_{3}$ direction on the two $\mathrm{d} A_{2}$ faces within the material and on a region.



(a)

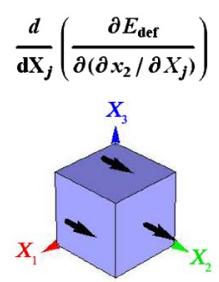

(b)

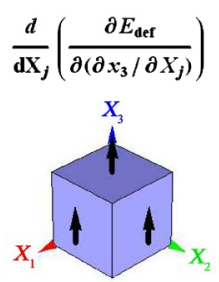

(c)

Figure 3. Forces in each direction on surfaces of cuboid (forces on the back sides not shown). (a) Surface forces in the $X_{1}$ direction; (b) Surface forces in the $X_{2}$ direction; (c) Surface forces in the $X_{3}$ direction. 
shown. There are forces on the rear surfaces that also contribute to each $F_{i}^{\text {net }}$ term.

\section{Some Details}

The procedure outlined in the last section to calculate the force on a plane after a deformation seems a bit convoluted in that the location of the plane before any deformation must be found in order to find the force on the plane after deformation. However, Equation (12) are excellent for applying Neumann boundary conditions to Equation (11). As an example, consider the case of deforming a rectangular body as shown in Figure 1(a) by applying some force on the $X_{3}$ face of the cuboid. If we know the components of the applied force from boundary conditions as a function of time, we can write

$$
F_{i}=\int \frac{\partial E_{d e f}}{\partial\left(\partial x_{i} / \partial X_{3}\right)} \mathrm{d} A_{3}
$$

If the force is applied uniformly over the area, $\mathrm{d} F_{i} / \mathrm{d} A_{3}$ is simply the applied force divided by a constant, the origial area. Therefore the Neumann boundary condition using Equation (12) is defined using just a rescaled version of the applied force on the surface of the material.

Finite deformations may displace and distorted planes in the cuboid from their original positions, but as long as inversions are not allowed, the same bounding surfaces of the cuboid are found regardless of how the material is deformed. The values of $\frac{\partial E_{\text {def }}}{\partial\left(\partial x_{i} / \partial X_{j}\right)}$ change from point to point as the material is deformed, but the $\mathrm{d} A_{j}$ vectors are unchanged by the deformation. Thus the forces shown in Figures 1-3 may be displaced due to the finite deformation, but the orientation of each component of each force from each surface is the same and the form of the sum of the forces, $\frac{\mathrm{d}}{\mathrm{d} X_{j}} \frac{\partial E_{d e f}}{\partial\left(\partial x_{i} / \partial X_{j}\right)}$, is unchanged by the displacement.

Lastly, it is tempting to consider the second order tensor quantity $\frac{\partial E_{d e f}}{\partial\left(\partial x_{i} / \partial X_{j}\right)}$ to be stress, but it is only stress for infinitesimal deformations. This is because $\frac{\partial E_{d e f}}{\partial\left(\partial x_{i} / \partial X_{j}\right)}$ must be multiplied by the ORIGINAL surface vector, not the current one to get the force at the current location.

\section{Conclusion}

The equations for dynamics in Euler-Lagrange elasticity have been derived. These equations are shown to be a simple statement of Newton's Law $\left(\sum \boldsymbol{F}=\frac{\mathrm{d} \boldsymbol{p}}{\mathrm{d} t}\right)$ for each infinitesimal volume of the material. The derived equations, Equation (11), are applicable to infinitesimal and finite deformations for both isotropic and anisotropic materials.

\section{References}

[1] Maugin, G.A. (2013) Continuum Mechanics through the Twentieth Century. Springer, London. http://dx.doi.org/10.1007/978-94-007-6353-1

[2] Truesdell, C. and Noll, W. (2009) The Non-Linear Field Theories of Mechanics. Springer, London.

[3] Srinivasa, A.R. and Srinivasan, S.M. (2004) Inelasticity of Materials. World Springer, New York.

[4] Pedregal, P. (2000) Variational Methods in Nonlinear Elasticity. Siam, Philadelphia.

[5] Hardy, H.H. (2013) Euler-Lagrange Elasticity: Differential Equations for Elasticity without Stress or Strain. Journal of Applied Mathematics and Physics, 1, 26-30.

[6] Todhunter, I. (1886) A History of the Theory of Elasticity and of the Strength of Materials from Galileo to the Present Time. Vol. 1, Cambridge University Press, New York.

[7] Shabana, A.A. (2008) Computational Continuum Mechanics. Cambridge University Press, New York, 131. http://dx.doi.org/10.1017/CBO9780511611469.005 
[8] Spencer, A.J. (1980) Continuum Mechanics. Dover, Mineola, New York.

[9] Ogden, R.W. (1984) Non-Linear Elastic Deformations. Dover, Mineola, New York.

[10] Hardy, H.H. and Shmidheiser, H. (2011) A Discrete Region Model of Isotropic Elasticity. Mathematics and Mechanics of Solids, 16, 317-333. http://dx.doi.org/10.1177/1081286510391666 
Scientific Research Publishing (SCIRP) is one of the largest Open Access journal publishers. It is currently publishing more than 200 open access, online, peer-reviewed journals covering a wide range of academic disciplines. SCIRP serves the worldwide academic communities and contributes to the progress and application of science with its publication.

Other selected journals from SCIRP are listed as below. Submit your manuscript to us via either submit@scirp.org or Online Submission Portal.
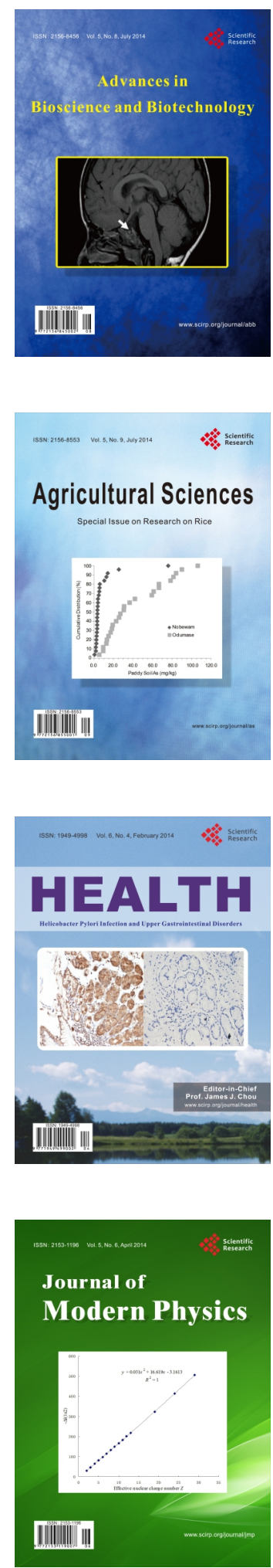
